\title{
Dépression atmosphérique sur le système de santé en Suisse
}

\section{Remo Osterwalder}

Dr med., membre du Comité central de la FMH, responsable du département Médecins en libre pratique

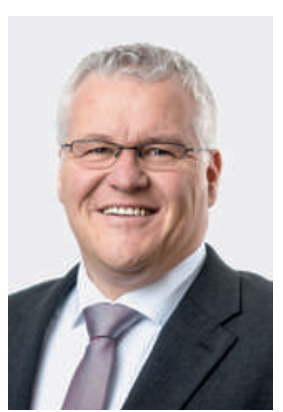

Ce qui se lit comme un message météo se profile également à l'horizon de notre système de santé. Si 2015 a été l'année la plus chaude depuis le début des mesures, on peut se demander ce que 2016 nous réserve. La situation concernant les thématiques du corps médical à moyen terme est cependant plus précise que les prévisions météorologiques annuelles. Une étape essentielle de l'année sera assurément la révision tarifaire qui mérite sans aucun doute le dénominatif de tâche herculéenne. Mais qui donc a jeté la pomme de la discorde et attend confortablement la suite? Après douze ans, il est non seulement légitime mais aussi nécessaire d'évaluer un tarif qui constitue le fondement de la représentativité de notre travail. Or, on a tout bonnement omis de définir un instrument de révision lors de l'introduction de la structure tarifaire. Alors que le contrôle régulier des véhicules est une évidence pour la plupart des automobilistes que nous sommes, un tel système ne devrait pas être contesté pour notre modèle de rémunération. Aux politiques, rappelons clairement que le service ne donne pas droit à un plein de carburant. En d'autres termes, il ne faut pas mélanger les questions du tarif et de prix. Le corps médical doit être sur ses gardes pour éviter que nous voguions entre les Symplégades.

Après douze ans, il est non seulement légitime mais nécessaire d'évaluer un tarif: le fondement de la représentativité de notre travail.

Nous entendons régulièrement le terme d'explosion des coûts; en géologie, une telle expression caractériserait une éruption volcanique. Simplement, les géologues ont en matière de prévisions un avantage considérable. Si, dans leur domaine, ils ne peuvent pas donner de meilleures prévisions que nous, médecins, pour l'évolution des coûts, ils savent en revanche que le lieu d'une éruption est clairement localisé et ne peut être contesté. Loin de moi l'idée de vouloir porter des accusations, il s'agit avant tout de se livrer à une analyse objective. Prenons donc un exemple: il est possible que la radiographie conventionnelle ne soit médicalement plus adaptée (guidelines) pour évaluer une maladie, mais il peut aussi arriver que les assureurs exigent une IRM en raison de la qualité des clichés pour évaluer leur devoir de prestation. Les deux cas sont tout à fait compréhensibles. Cependant, cet exemple nous dit aussi qu'on ne peut pas parler de manière non différenciée de l'explosion des coûts. Comment agir contre l'hétérogénéité de l'évolution des coûts? La densité de médecins doit-elle être régulée par l'Etat ou appartient-il aux partenaires de chercher ensemble des instruments efficaces? Est-ce que dans ce cas, une graduation du prix en fonction de la région et de la qualité, comme l'exige la motion 15.3385 [1] du PLR, peut être efficace?

En 2015, un orage inattendu, annoncé initialement comme une légère averse, nous a surpris au sommet du gel des admissions.

L’année dernière, un orage inattendu, annoncé initialement comme une légère averse, nous a surpris au sommet du gel des admissions. Nous n'étions équipés ni de bottes ni de paratonnerre. La réaction ne s'est pas fait attendre avec la proposition du gouvernement vaudois de reprendre la discussion en faveur des caisses-maladie cantonales, sans clarifier s'il s'agit du monopole d'une institution ou d'une alternative. Cette question avait été anticipée lors de mes réflexions dans un précédent éditorial [2].

D’autres turbulences sont attendues, notamment avec la motion 13.3265, "Contre-proposition à la limitation de l'admission de médecins», qui prévoit l'introduction de la liberté de contracter à partir d'une certaine densité médicale. Est-ce une solution pragmatique et administrativement supportable? Qui définirait le seuil critique et selon quels critères? Cela renforcerait-il l'influence du corps médical au travers des sociétés cantonales de médecine? N'est-il pas surprenant de trouver autant de similitudes avec nos collègues météorologues? Alors que l'hiver semble ne pas vouloir venir, espérons qu'aucune période de glaciation ne s'installe.

1 Système de santé. Equilibrer l'offre de soins en différenciant la valeur du point tarifaire au lieu de geler une nouvelle fois les admissions. www.parlament.ch/f/suche/Pages/geschaefte.aspx?gesch_ id $=20153385$.

2 Osterwalder R. Une votation peut en cacher une autre. Bull Méd Suisses. 2015;96(3):49. 